\title{
Antithrombotic treatment with direct-acting oral anticoagulants in patients with splanchnic vein thrombosis and cirrhosis
}

\begin{tabular}{|c|c|}
\hline Andrea De Go & ottardi $^{1,{ }^{*}}$ | Jonel Trebicka ${ }^{2, *}$ | Christoph Klinger ${ }^{3}$ | Aurélie Plessier ${ }^{4}$ | \\
\hline Susana Seijo ${ }^{5}$ & | Benedetta Terziroli ${ }^{6} \mid$ Lorenzo Magenta $^{6} \mid$ David Semela $^{7} \mid$ Elisabetta \\
\hline Buscarini $^{8}$ | & Philippe Langlet $^{9}$ | Jan Görtzen ${ }^{2}$ | Angela Puente ${ }^{10}$ | Beat Müllhaupt ${ }^{11}$ | \\
\hline Carmen Nava: & scuès $^{12} \mid$ Filipe Nery $^{13} \mid$ Pierre Deltenre $^{14} \mid$ Fanny Turon $^{15} \mid$ Cornelius \\
\hline Engelmann ${ }^{16}$ & | Rupen Arya ${ }^{5}$ | Karel Caca ${ }^{3}$ | Markus Peck-Radosavljevic ${ }^{17}$ | Frank W. \\
\hline & Dominique Valla $^{4}$ | Juan Carlos Garcia-Pagan ${ }^{15} \mid$ on behalf of the VALDIG \\
\hline
\end{tabular}

${ }^{1}$ Hepatology, Department of Clinical Research and Clinic of Visceral Surgery and Medicine, Inselspital, University of Berne, Berne, Switzerland

${ }^{2}$ University Hospital, Bonn, Germany

${ }^{3}$ Klinikum Ludwigsburg, Ludwigsburg, Germany

${ }^{4} \mathrm{DHU}$ Unit, Hôpital Beaujon, Clichy-laGarenne, France

${ }^{5}$ King's College, London, UK

${ }^{6}$ Epatocentro Ticino, Lugano, Switzerland

${ }^{7}$ Kantonsspital St. Gallen, St. Gallen, Switzerland

${ }^{8}$ Maggiore Hospital, Crema, Italy

${ }^{9} \mathrm{CHIREC}$, Brussels, Belgium

${ }^{10} \mathrm{HUMV}$ and Investigation Institute

Marques de Valdecilla, Santander, Spain

${ }^{11}$ University Hospital, Zürich, Switzerland

${ }^{12}$ Hospital Universitario de Asturias, Oviedo, Spain

${ }^{13}$ Hospital Santo Antonio, Porto, Portugal

${ }^{14} \mathrm{CHUV}$, Lausanne, Switzerland

${ }^{15}$ Hospital Clinic, IDIBAPS and CIBERehd, Barcelona, Spain

${ }^{16}$ University Hospital Leipzig, Leipzig, Germany

${ }^{17}$ Medical University, Vienna, Austria

${ }^{18}$ Department of Hematology, Erasmus University Medical Center, Rotterdam, The Netherlands

\begin{abstract}
Background: Direct-acting oral anticoagulants (DOACs) are used in patients with splanchnic vein thrombosis (SVT) and cirrhosis, but evidence for safety and efficacy in this setting is limited. Our aim was to identify indications and reasons for starting or switching to DOACs and to report adverse effects, complications and short-term outcome.

Methods: Data collection including demographic information, laboratory values, treatment and complications through the Vascular Liver Disease Interest Group Consortium. Results: Forty-five centres (90\%) of the consortium completed the initial eCRF. We report here a series of 94 patients from 17 centres. Thirty-six patients (38\%) had cirrhosis. Child-Pugh score was 6 (range 5-8), and MELD score 10.2 (range 6-19). Indications for anticoagulation were splanchnic vein thrombosis (75\%), deep vein thrombosis (5\%), atrial fibrillation (14\%) and others (6\%). DOACs used were rivaroxaban (83\%), dabigatran (11\%) and apixaban (6\%). Patients were followed up for a median duration of 15 months (cirrhotic) and 26.5 months (non-cirrhotic). Adverse events occurred in $17 \%$ of patients and included one case of recurrent portal vein thrombosis and five cases of bleeding. Treatment with DOACs was stopped in three cases. The major reasons for choosing DOACs were no need for monitoring or inadequacy of INR to guide anticoagulation in cirrhotic patients. Renal and liver function did not change during treatment.

Conclusions: A consistent number of patients with SVT and/or cirrhosis are currently treated with DOACs, which seem to be effective and safe. These data provide a basis for performing randomized clinical trials of DOACs vs. low molecular weight heparin or vitamin $\mathrm{K}$ antagonists.
\end{abstract}


Correspondence

Andrea De Gottardi, Hepatology,

Department of Clinical Research and Clinic

of Visceral Surgery and Medicine, Inselspital,

University of Berne, Berne, Switzerland.

Email: andrea.degottardi@gmail.com

Handling Editor: Christophe Bureau

\section{KEYWORDS}

anticoagulation, Budd-Chiari syndrome, cirrhosis, portal vein thrombosis

\section{1 | INTRODUCTION}

Direct-acting oral anticoagulants (DOACs) are currently approved for the prevention of ischaemic stroke in patients with atrial fibrillation and as treatment of deep venous thrombosis of the leg and pulmonary embolism. ${ }^{1}$ However, they have also been used "off label" in several other indications, including portal vein thrombosis and Budd-Chiari syndrome in a limited number of patients, ${ }^{2-4}$ but their efficacy and safety in these situations remain unknown.

In addition, the use of concomitant medications, age and comorbidities, renal insufficiency and impairment of liver function, may significantly affect pharmacokinetics and pharmacodynamics of DOACs. ${ }^{5,6}$ In this context, patients with advanced liver disease raise a double challenge primarily due to the known derangements of the coagulation system, ${ }^{7}$ and secondarily due to the potential impact of the impairment of the liver function on the efficacy and safety of DOACs. This is especially relevant because of the nowadays confirmed high prevalence of thrombotic events in patients with cirrhosis with the ensuing increase in the use of anticoagulants., ${ }^{8,9}$

The scarcity of data on the use of DOACs for the treatment of splanchnic vein thrombosis ${ }^{10}$ and/or in patients with cirrhosis ${ }^{11}$ as well as recent reported cases of direct DOAC-related hepatotoxicity ${ }^{12}$ prompted us to perform this survey. In recent publication reporting data from 20 cirrhotic patients treated with DOACs the authors did not identify any increased risks compared to patients treated with vitamin $\mathrm{K}$ antagonists or $\mathrm{LMWH}{ }^{13}$

We set out to collect information about the indications and the reasons for starting or switching to DOACs in patients with cirrhosis and/or splanchnic vein thrombosis and to describe possible adverse effects and safety issues in this particular group of patients with portal hypertension.

\section{2 | MATERIALS AND METHODS}

A survey using an electronic e-CRF was performed initially between February and December 2014 followed by a second data collection between April and July 2016 among the members of the VALDIG Consortium (www.valdig.eu) to gather information about the use of DOACs in patients with cirrhosis and/or splanchnic vein thrombosis (Table S1).

There were two inclusion criteria: (i) the use of DOACs (rivaroxaban, apixaban, dabigatran), and (ii) the presence of cirrhosis (based on histology or clinical and imaging data) and/or splanchnic vein thrombosis (including portal vein thrombosis or Budd-Chiari syndrome).

\section{Key points}

- Direct-acting oral anticoagulants are used for the prevention or treatment of several thrombotic disorders.

- Data about safety and efficacy of these drugs in patients with cirrhosis and/or splanchnic vein thrombosis are scarce.

- Here, we report the use of DOACs in 94 patients from 17 European centres.

- Results on effectiveness and safety are of DOACs in this patient population provide the basis for randomized clinical trials.

Patient's initial information was recorded at the time of starting DOACs followed by data collection at the two time points indicated above.

Adverse events were recorded including hepatotoxicity, gastrointestinal symptoms and bleeding of any grade. Major bleeding was defined by the presence of at least one of the following characteristics: need for hospitalization, or transfusion of at least two units of packed red blood cells, or intracranial or retroperitoneal location or leading to death.

Data analysis was performed using descriptive statistics and comparisons between laboratory values were performed using Mann-Whitney test. A $P$ value of $<.05$ was considered statistically significant.

According to a position statement of the ethic committee of the University Hospital of Bern, Switzerland (date 27 January 2014), patients' approval was not mandatory since this data collection was fully anonymized.

\section{3 | RESULTS}

Among the 50 centres belonging to the VALDIG Consortium during the period of this study, 45 (90\%) provided data concerning the use of DOACs in patients with cirrhosis and/or splanchnic vein thrombosis. Of the 45 participating centres, 28 did not report any patients fulfilling the inclusion criteria, while 17 centres provided data about 94 patients treated with DOACs. The baseline characteristics of this study population, the indication for anticoagulation and the anticoagulant used are summarized in Table 1.

The daily dose of DOACs was determined by the local investigators and there was no standardized adjustment across the centres. In 
patients with decompensated cirrhosis DOACs were not used, while in Child-Pugh A cirrhosis each investigator determined the dosage on a case-by-case basis. ${ }^{14}$

\section{1 | Patients without cirrhosis}

Fifty-eight patients with splanchnic vein thrombosis without underlying cirrhosis receiving DOACs were identified. Portal vein thrombosis and cardiac arrhythmia were the main indications for anticoagulation in $65 \%$ and $21 \%$ of these non-cirrhotic patients respectively. DOACs were the initial anticoagulant in $34 \%$ of patients, while the remaining $66 \%$ of cases were previously treated with another anticoagulant (the most frequently used anticoagulants were low molecular weight heparins, followed by vitamin $\mathrm{K}$ antagonists including phenprocoumon, warfarin and acenocoumarol). The main reasons for switching to DOACs were development of recurrent thrombosis, INR instability and clinically relevant side effects. In one-third of the patients, no need of INR monitoring was the reason to choose DOACs. Other reasons included patient's comfort and no need for injections in $10 \%$ and $7 \%$ respectively.
In the initial data collection, the duration of treatment with DOACs was 13.1 (mean) and 10.5 (median) months (range 2-48). In this period, adverse events were reported in $11(19 \%)$ of patients (Figure 1A).

No cases of mortality related to the use of DOACs were reported during this study period. The median daily dose of all DOACs (i.e. rivaroxaban, apixaban and dabigatran) in patients without cirrhosis was $25 \%$ higher than in patients with cirrhosis (Table 1).

Follow-up data were available for 26 patients who were treated with DOACs for a total duration of 27.9 (mean) and 26.5 (median) months. Adverse events were newly reported in five cases including three haemorrhagic complications and two thrombotic events. Haemorrhages occurred from oesophageal varices, from a gastric ulcer and in one case in the form of a prolonged menstrual bleeding. Anticoagulation therapy was continued, but in two cases, it was changed for low molecular weight heparin. The two thrombotic events included one case of progression of portal vein thrombosis, for which rivaroxaban was replaced by phenprocoumon and one case of obstruction of a meso-caval shunt in which rivaroxaban was stopped and a treatment with LMWH was introduced.

\begin{tabular}{|c|c|c|}
\hline & Patients without cirrhosis $(n=58)$ & Patients with cirrhosis ( $n=36$ ) \\
\hline Age $(Y)$ & 49.5 (range 16-82) & 64.9 (range 32-82) \\
\hline Gender & 29 F-29 M (50\%-50\%) & 15 F-21 M (42\%-58\%) \\
\hline \multicolumn{3}{|l|}{ Aetiology of cirrhosis } \\
\hline Alcohol & - & $10(28 \%)$ \\
\hline $\mathrm{HCV}$ & - & $6(17 \%)$ \\
\hline NAFLD/NASH & - & $8(22 \%)$ \\
\hline Other & - & $12(33 \%)$ \\
\hline \multicolumn{3}{|c|}{ Oesophageal or gastric varices: } \\
\hline Total & $24(41 \%)$ & $23(64 \%)$ \\
\hline Large & $15(62 \%)$ & 7 (30\%) \\
\hline $\begin{array}{l}\text { Previous variceal } \\
\text { bleeding }\end{array}$ & 17 (70\%) & $9(39 \%)$ \\
\hline Presence of ascites & $13(22 \%)$ & $12(33 \%)$ \\
\hline \multicolumn{3}{|c|}{ Indication for anticoagulation } \\
\hline $\begin{array}{l}\text { Portal vein } \\
\text { thrombosis }\end{array}$ & $38(65 \%)$ & $22(61 \%)$ \\
\hline $\begin{array}{l}\text { Budd-Chiari } \\
\text { Syndrome }\end{array}$ & $4(7 \%)$ & $5(14 \%)$ \\
\hline Cardiac arrhythmia & $12(21 \%)$ & 5 (14\%) \\
\hline $\begin{array}{l}\text { Peripheral deep vein } \\
\text { thrombosis }\end{array}$ & - & $2(5 \%)$ \\
\hline Other & $6(10 \%)^{a}$ & $2(5 \%)^{b}$ \\
\hline \multicolumn{3}{|c|}{ DOAC used and median daily dose } \\
\hline Rivaroxaban & 49 (84\%), 20 mg (range 10-20 mg) & 30 (83\%), 15 mg (range 5-20 mg) \\
\hline Apixaban & 6 (10\%), 7.5 mg (range 5-10 mg) & 4 (11\%), 5 mg (range $2.5-10 \mathrm{mg}$ ) \\
\hline Dabigatran & 3 (5\%), 220 mg (range 150-220 mg) & 2 (5\%), $165 \mathrm{mg}$ (range $110-220 \mathrm{mg}$ ) \\
\hline
\end{tabular}

TABLE 1 Characteristics of this study population

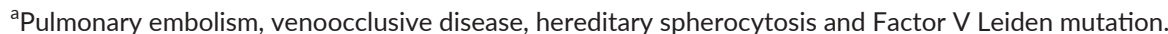

${ }^{\mathrm{b}}$ Peripheral deep vein thrombosis. 


\section{2 | Patients with cirrhosis}

Thirty-six patients with cirrhosis received DOACs. The causes of cirrhosis are summarized in Table 1. The main indication for DOACs was portal vein thrombosis (61\%), concomitant Budd-Chiari Syndrome (14\%), cardiac arrythmia (14\%), followed by deep vein thrombosis (5\%) and others (5\%). The majority of these patients (58\%) was initially treated with other anticoagulants.

According to investigators survey, in one-third of the patients, a switch from vitamin $\mathrm{K}$ antagonists to DOACs was deemed justified by the fact that, when spontaneously prolonged due to cirrhosis, INR would be inappropriate for monitoring anticoagulation. Other reasons for switching to DOACs included INR instability or the development of side effects (14\%), patient's comfort (14\%), no need for INR monitoring or no need for injections (16\%).

The mean duration of treatment with DOACs in the initial data collection was 9.6 (mean) and 7.0 (median) months (range 2-48). In this period, adverse events were reported in $6(17 \%)$ of patients (Figure 1B).
At the beginning of treatment, the mean Child-Pugh score was 6 (range 5-8) and remained unchanged after DOACs. Similarly, the MELD score did not change significantly from 10.2 to 11.3 points. In both cirrhotic and non-cirrhotic patients, liver and renal function tests did not significantly change during the treatment with DOACs (Table 2). DOAC serum levels or factor Xa were not routinely monitored.

Patients with cirrhosis were prescribed more often with a reduced dose of DOACs (23 out of 36 patients) than those without cirrhosis (17 of 58 patients) and this difference was statistically significant ( $P=.00098$ using Chi-Square test).

Follow-up data were available for 18 patients with cirrhosis, who were treated with DOACs for a total duration of 14.6 (mean) and 15.0 (median) months. During this period, one patient died of pneumonia and another one was lost to follow-up. Treatment with DOACs was stopped in five patients. In two patients DOACs were stopped and replaced by LMWHs after lower gastrointestinal bleeding. In three patients, anticoagulation was definitively stopped after the development of hepatorenal syndrome followed by liver transplantation in one case and after TIPS insertion in two patients.

(A)

\section{Patients without cirrhosis $n=58$}

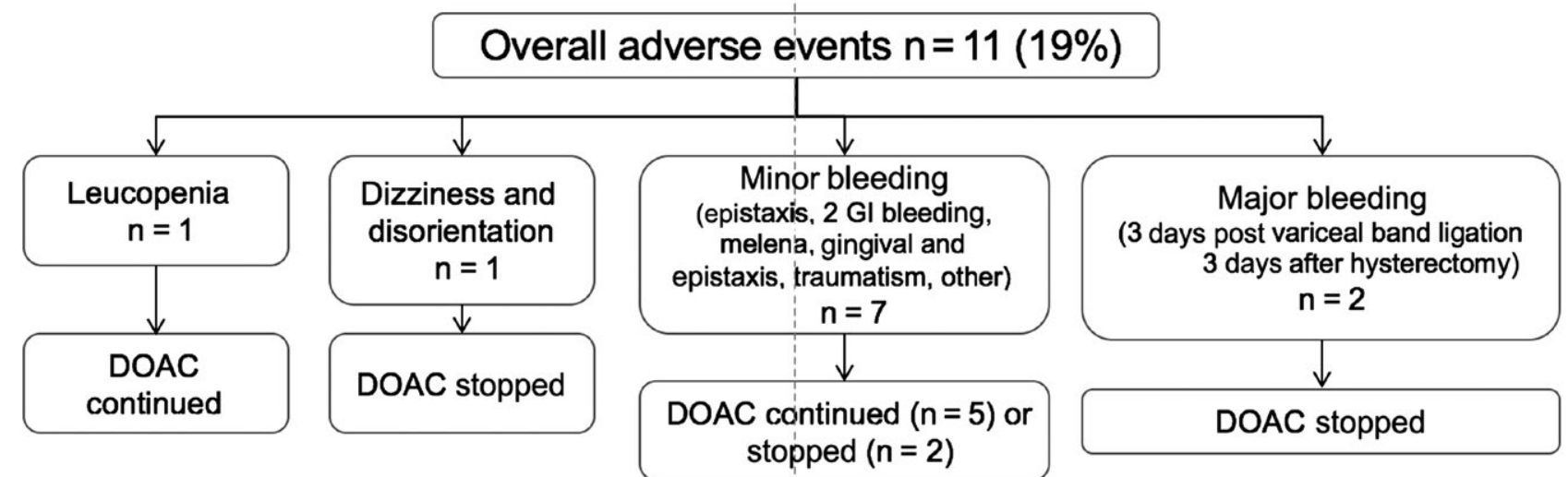

(B)

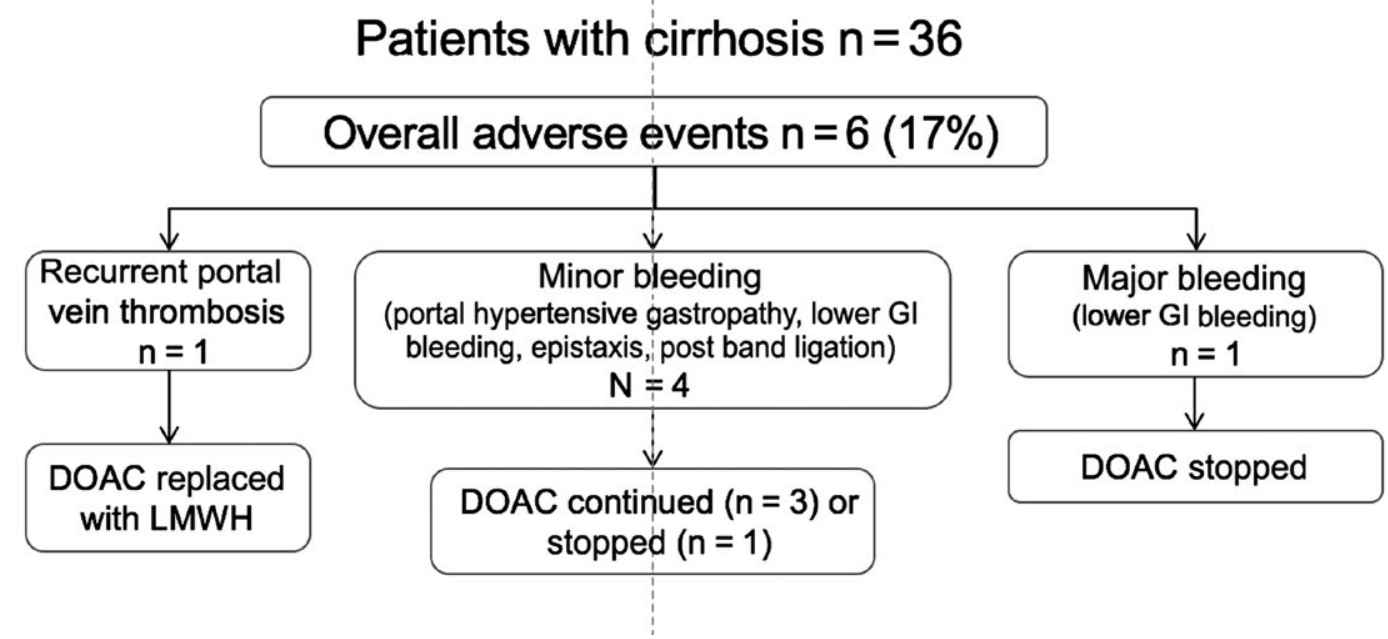

FIGURE 1 Overall incidence of adverse events reported with the use of DOACs in patients without cirrhosis (1A) or with cirrhosis (1B). The schemes describe the number of patients and the type of adverse events and whether DOACs were stopped as a consequence of these complications 
TABLE 2 Laboratory data of this study population

\begin{tabular}{|c|c|c|c|c|c|c|c|c|}
\hline & \multicolumn{4}{|c|}{ Patients without cirrhosis } & \multicolumn{4}{|c|}{ Patients with cirrhosis } \\
\hline Creatinine $(\mu \mathrm{mol} / \mathrm{L})$ & $71 \pm 24$ & $70 \pm 29$ & $80 \pm 50$ & ns & $77 \pm 24$ & $81 \pm 42$ & $97 \pm 28$ & ns \\
\hline INR & $1.20 \pm 0.29$ & $1.20 \pm 0.19$ & $1.19 \pm 0.34$ & ns & $1.14 \pm 0.20$ & $1.20 \pm 0.19$ & $1.17 \pm 0.20$ & ns \\
\hline Albumin (g/L) & $38 \pm 7$ & $39 \pm 6$ & $40 \pm 7$ & ns & $36 \pm 5$ & $35 \pm 6$ & $33 \pm 8$ & ns \\
\hline Thrombocytes G/L & $221 \pm 153$ & $200 \pm 153$ & $196 \pm 131$ & ns & $122 \pm 163$ & $126 \pm 121$ & $107 \pm 151$ & ns \\
\hline ALT (IU/L) & $29 \pm 49$ & $30 \pm 26$ & NA & ns & $43 \pm 35$ & $37 \pm 24$ & NA & ns \\
\hline
\end{tabular}

All data are expressed as median values \pm SD.

\section{DISCUSSION}

Despite the lack of registration for these indications, we show here that DOACs are used in patients with portal vein thrombosis, BuddChiari syndrome or cirrhosis in approximately $40 \%$ of the centres belonging to the VALDIG Consortium.

These data represent so far the largest available series on the use of DOACs in patients with splanchnic vein thrombosis or with impaired liver function due to cirrhosis. However, it must be stressed that no patient with advanced liver disease in the stage Child-Pugh $C$ was treated with DOACs. This is likely due to the current safety recommendations concerning the use of these drugs in patients with severely impaired liver function. ${ }^{15,16}$ In addition, in cirrhotic patients, all three DOACs were administered in a lower dose than the one administered to non-cirrhotic patients (Table 1 ) despite treating similar thrombotic conditions. Only 13 of 36 cirrhotic patients (36\%) in contrast to 41 of 58 non-cirrhotic patients $(71 \%)$ were prescribed a full anticoagulant dose. The lower dose used was not related to an adaptation of the dose to kidney dysfunction, since creatinine was similar in patients with and without cirrhosis and within the normal range. This could rather indicate that physicians were administering DOACs with particular caution in patients with cirrhosis. Thus, considering the limitation that cirrhotic patients were possibly undertreated and that no patients with Child-Pugh C cirrhosis were included, the experience reported here suggests that the use of DOACs can be extended to patients with Child-Pugh A and B stages. In their recent study, ${ }^{13}$ Intagliata et al. found no significant differences in terms of safety and efficacy in cirrhotic patients treated with DOACs when compared to cirrhotic patients under treatment with vitamin $\mathrm{K}$ antagonists or LMWH. Similar to this study, the authors did not include patients with advanced cirrhosis (stage Child-Pugh C).

On one hand, these findings increase the growing amount of clinical evidence that anticoagulation in general is not particularly dangerous in cirrhosis. On the other hand, they extend the armamentarium of anticoagulants for cirrhotic patients, by including DOACs in addition to $\mathrm{LMWH}$ and vitamin $\mathrm{K}$ antagonists. The benefit of using DOACs in patients with cirrhosis will remain, however, unclear until head to head comparison of traditional and new anticoagulants will be available. ${ }^{14}$
The incidence of complications, also including bleeding episodes, related to anticoagulation in this study was similar in patients with or without cirrhosis. Recurrence of thrombosis of the portal vein under treatment with rivaroxaban may indicate insufficient dosing or, as suggested by in vitro results, ${ }^{9}$ that the potency of this drug might be reduced in cirrhotic patients. Further studies, preferably randomized clinical trials including a large number of patients are needed to address this issue.

An increased risk of gastrointestinal bleeding with an incidence of $1.4 \%$ in patients treated with DOACs has been described in a recent meta-analysis. ${ }^{17}$ The four cases of major gastrointestinal bleeding out of 94 patients from our series correspond to an incidence of 5.1\% over a median duration of 26.5 months in patients without cirrhosis and of $2.7 \%$ over a median duration of 15 months in patients with cirrhosis. Data from a recent survey showed that in cirrhotic patients treated with DOACs for a mean duration of 8.9 months, the incidence of major bleedings was $5 \%{ }^{13}$ and that in a control group of patients treated with traditional anticoagulants the incidence of bleeding was similar. Additional studies showed that the incidence of minor and major gastrointestinal bleeding in cirrhotic patients treated with LMWH was $8 \%{ }^{18}$ and $9 \%$ over a follow-up of 19 months. ${ }^{19}$ These findings are at some variance with those reported earlier, in where no bleeding complications occurred in cirrhotic patients treated with traditional anticoagulants for 24 months. ${ }^{20}$ Therefore, based on the currently available evidence, the use of DOACs in cirrhotic patients seems to be as safe and effective, as the traditional anticoagulants, provided that liver function is within Child-Pugh stages A or B. It is however obvious that more data are needed to better assess the risk/benefit ratio of DOACs in this group of patients.

Hepatotoxicity of DOACs has been reported previously ${ }^{12,21,22}$ and is certainly a point of concern in particular in patients with chronic advanced liver disease. Nevertheless, in the 94 patients of this study, no hepatotoxicity was observed. Since the incidence of DOACs-induced liver toxicity is estimated to be less than $1 \%$, the number of patients included in this survey is not sufficiently representative to confirm these data.

It must be stressed that caution is needed for the interpretation of the data presented here due to the limitations of this study design. For example, the lack of a control group including patients treated with traditional anticoagulants allowing a comparison represents a significant drawback. 
In conclusion, while these results do not allow any recommendations on the use of DOACs in patients with splanchnic vein thrombosis and/or cirrhosis, they indicate that these agents are already used off label in this population. These observational data provide a basis for building randomized clinical trials of DOACs vs. LMWH or vitamin $\mathrm{K}$ antagonists in these patient groups.

\section{ACKNOWLEDGEMENTS}

The authors would like to thank all members of the VALDIG Consortium and investigators, who have contributed to this survey (appendix). In addition, we would like to thank the European Association for the Study of the Liver (EASL) and the REHEVASC group of the Asociación Española para el Estudio del Hígado for supporting VALDIG.

\section{CONFLICT OF INTEREST}

The authors declare that there are no conflicts of interests.

\section{REFERENCES}

1. Gómez-Outes A, Suárez-Gea ML, Lecumberri R, Terleira-Fernández Al, Vargas-Castrillón E. Direct-acting oral anticoagulants: pharmacology, indications, management, and future perspectives. Eur J Haematol. 2015;95:389-404.

2. Martinez M, Tandra A, Vuppalanchi R. Treatment of acute portal vein thrombosis by nontraditional anticoagulation. Hepatology. 2014;60:425-426

3. Pannach S, Babatz J, Beyer-Westendorf J. Successful treatment of acute portal vein thrombosis with rivaroxaban. Thromb Haemost. 2013;110:626-627.

4. Lenz K, Dieplinger B, Buder R, Piringer P, Rauch $M$, Voglmayr $M$. Successful treatment of partial portal vein thrombosis (PVT) with low dose rivaroxaban. Z Gastroenterol. 2014;52:1175-1177.

5. DeWald TA, Becker RC. The pharmacology of novel oral anticoagulants. J Thromb Thrombolysis. 2014;37:217-233.

6. Mavrakanas TA, Samer C, Fontana P, Perrier A. Direct oral anticoagulants: efficacy and safety in patient subgroups. Swiss Med Wkly. 2015;145:w14081.

7. Tripodi A, Mannucci PM. The coagulopathy of chronic liver disease. N Engl J Med. 2011;365:147-156.

8. Graff J, Harder S. Anticoagulant therapy with the oral direct factor $\mathrm{Xa}$ inhibitors rivaroxaban, apixaban and edoxaban and the thrombin inhibitor dabigatran etexilate in patients with hepatic impairment. Clin Pharmacokinet. [Internet]. 2013;52:243-254. Available from: http:// www.ncbi.nlm.nih.gov/pubmed/23389892

9. Potze W, Adelmeijer J, Lisman T. Decreased in vitro anticoagulant potency of Rivaroxaban and Apixaban in plasma from patients with cirrhosis. Hepatology. 2015;61:1435-1436.

10. Ageno W, Riva N, Schulman S, et al. Long-term clinical outcomes of splanchnic vein thrombosis: results of an international registry. JAMA Intern Med. 2015;175:1474-1480.

11. Intagliata NM, Maitland H, Northup PG, Caldwell SH. Treating thrombosis in cirrhosis patients with new oral agents: ready or not? Hepatology. 2015;61:738-739.

12. Russmann S, Niedrig DF, Budmiger M, et al. Rivaroxaban postmarketing risk of liver injury. J Hepatol. 2014;61:293-300.
13. Intagliata NM, Henry ZH, Maitland $\mathrm{H}$, et al. Direct oral anticoagulants in cirrhosis patients pose similar risks of bleeding when compared to traditional anticoagulation. Dig Dis Sci. 2016;61:1721-1727.

14. Intagliata NM, Maitland H, Caldwell SH. Direct oral anticoagulants in cirrhosis. Curr Treat Options Gastroenterol. 2016;14:247-256.

15. Tran H, Joseph J, Young L, et al. New oral anticoagulants: a practical guide on prescription, laboratory testing and peri-procedural/ bleeding management. Intern Med J. [Internet]. 2014 [cited 2015 Sep 2];44:525-536. Available from: http://onlinelibrary.wiley.com/ doi/10.1111/imj.12448/abstract

16. Lisman T, Kamphuisen PW, Northup PG, Porte RJ. Established and new-generation antithrombotic drugs in patients with cirrhosis Possibilities and caveats. J Hepatol. [Internet]. 2013 [cited 2015 Aug 16];59:358-366. Available from: http://www.journal-of-hepatology. eu/article/S0168827813002067/abstract

17. Holster IL, Valkhoff VE, Kuipers EJ, Tjwa ETTL. New oral anticoagulants increase risk for gastrointestinal bleeding: a systematic review and meta-analysis. Gastroenterology. 2013;145:105-112.e15.

18. Bechmann LP, Sichau M, Wichert M, Gerken G, Kröger K, Hilgard P. Low-molecular-weight heparin in patients with advanced cirrhosis. Liver Int. 2011;31:75-82.

19. Delgado MG, Seijo S, Yepes I, et al. Efficacy and safety of anticoagulation on patients with cirrhosis and portal vein thrombosis. Clin Gastroenterol Hepatol. 2012;10:776-783.

20. Amitrano L, Guardascione MA, Menchise A, et al. Safety and efficacy of anticoagulation therapy with low molecular weight heparin for portal vein thrombosis in patients with liver cirrhosis. J Clin Gastroenterol. 2010;44:448-451.

21. Liakoni E, Bravo AER, Krähenbühl S. Hepatotoxicity of New Oral Anticoagulants (NOACs). Drug Saf. [Internet]. 2015 [cited 2015 Aug 21];38:711-720. Available from: http://link.springer.com/ article/10.1007/s40264-015-0317-5

22. Liakoni $E$, Rätz Bravo $A E$, Terracciano L, Heim M, Krähenbühl S. Symptomatic hepatocellular liver injury with hyperbilirubinemia in two patients treated with rivaroxaban. JAMA Intern Med. 2014:174:1683-1686.

\section{SUPPORTING INFORMATION}

Additional Supporting Information may be found online in the supporting information tab for this article.

\section{APPENDIX}

\section{Vascular Liver Disease Interest Group (VALDIG)}

Henning Grønbæk, Aarhus, Jürg-Hans Beer, Baden, Joan Genescà, Barcelona, Canete Hidalgo, Barcelona, Carmen Vila, Barcelona, Jelena Mihailovic, Bern, Fabio Piscaglia, Bologna, Luc Lasser, Brussels, Miguel Fernandez-Bermejo, Caceres, Bogdan Procopet, Cluj, Jeoffrey Schouten, Ghent, Laurent Spahr, Geneva, José Louis Sanchez, Granada, Alexander Zipprich, Halle, Mallika Sekhar, London, Manuel Hernandez-Guerra, La Laguna, Frederik Nevens, Leuven, Carlos Noronha Ferreira, Lissabon, Michael Heneghan, London, Agustin Albillos, Madrid, Elba Llop, Madrid, Alessandra Dell'Era, Milano, Vincenzo La Mura, Milano, Massimo Primignani, Milano, Marco Senzolo, Padova, Ana Escarda, Palma de Mallorca, Oliviero Riggio, Roma, Sarwa Darwish Murad, Rotterdam, Stephan Haas, Stockholm, Christophe Bureau, Toulouse, Marina Berenguer, Valencia. 